\title{
Introducing e-developers to support a university's blended learning developments
}

\author{
Ann Ooms ${ }^{a}$, Linda Burke ${ }^{b *}$, Tim Linsey ${ }^{\mathrm{a}}$ and Celayne Heaton-Shrestha ${ }^{\mathrm{c}}$ \\ ${ }^{a}$ Academic Development Centre, Kingston University, UK; ${ }^{b}$ Faculty of Health and Social Care \\ Sciences, Kingston University and St George's, University of London, UK; ${ }^{c}$ Department of \\ Anthropology, University of Sussex, UK
}

\begin{abstract}
Introducing technology in higher education raises questions about staff roles and the organisation of development practices. This article presents the findings from a case study that was undertaken to evaluate the effectiveness of introducing three centrally supported e-developers to work with academic teams to provide specialist support. The e-developer role is explained, and related to existing literature about learning technologists. The case illustrates how the e-developers worked collaboratively with academic staff and the perceptions of the academic staff, e-developers and educational technology leaders of the e-developer model used in a university in southwest London. The findings offer an opportunity to understand this kind of role, and the value of a model of staff development that does not involve taking academic staff out of the teaching area to become e-developers. The model supports 'situative' professional development, which helps promote technology integration into teaching and suggests that e-developers provided cost-effective mentorship which participants believed would have a positive impact on student learning.
\end{abstract}

Keywords: blended learning; curriculum development; e-learning; learning resources; professional development; strategy; technology

\section{Background}

\section{Introduction}

This article presents a case study that was undertaken to measure the effectiveness of introducing three centrally provided e-developers to support academic staff in developing blended learning.

The aims of the research were to assess the effectiveness of the e-developer model employed in one university in terms of engagement of staff with blended learning and dissemination of good practice. An additional project aim was to assess the sustainability of the developments initiated by the e-developers in terms of their ongoing maintenance and development by the course teams they supported and worked alongside.

\section{Literature review}

Three strands of literature were identified for this literature review: the impact of technology on learning; support for blended learning; and professional/staff development for academic staff and for technology support staff.

\footnotetext{
*Corresponding author. Email: 1mburke@btinternet.com
} 
There is a growing literature related to the positive impact of technology on learning in schools, colleges and universities, the need for appropriate staff development for both educators and learning technologists and the emerging and diverse roles of learning technologists and how they support educators.

When applied to well-defined educational objectives, and integrated into the curriculum by trained teachers, education technology can produce dramatic results for students. (CEO Forum School Technology 2001, 6)

Researchers have shown that new instructional approaches are necessary to enable technology integration (Borko 2004; Zhao et al. 2002) because the use of technology does not automatically impact on faculty members' overall approach to teaching (Cuban 2000). Therefore, as teachers and lecturers make extensive use of technology, they need to confront the tension between traditional and new pedagogic methods.

On average, educational institutions in the UK invest $5.6 \%$ of their total budget in technology (UCISA 2006); however, for that investment to be returned, they also need to commit to professional development by providing the required support, resources and time for teachers to learn how to use technology. Several essential elements of professional development to support integration of technology have been identified in previous research. One of those essential elements is release time (Pajo and Wallace 2001; Thurlow 1999). Educators are expected to learn both technical skills and pedagogical strategies, and that requires an enormous amount of time. The issue of insufficient time is a major barrier for educators' use of technology because of contextual factors such as the structure of timetables and workload (Cuban, Kirkpatrick, and Peck 2001; Pajo and Wallace 2001; O'Neill, Singh, and O'Donoghue 2004), as well as the competing demand of time in terms of teaching versus research.

Several researchers have identified access to hardware and software at home (Adamy 2000; Cathings and MacGregor 2000; Norum, Grabinger, and Duffield 1999; Thurlow 1999) and in the institution (Thurlow 1999) as other essential elements for effective professional development. Official recognition of efforts to integrate technology is important as well. This can take on multiple formats, such as providing financial incentives and rewards, and provision of education credits and certification endorsement (Pajo and Wallace 2001; Thurlow 1999). Technical support for troubleshooting technical problems as they arise (Adamy 2000; Pajo and Wallace 2001; Thurlow 1999) is yet another essential element of support, as is follow-up training and ongoing mentor support (Chuang, Thompson, and Schmidt 2003; Cole, Simkins, and Penul 2002; Pajo and Wallace 2001; Polselli 2002; Sugar 2005; Swan et al. 2002; Thurlow 1999).

A critical factor for the effective implementation of e-learning in universities is the development of academic staff. Bates (2000) suggests that faculty development seems to work best when supported by a range of strategies, including staff incentives, staff support and professional development. Kenny (2002) suggests that support required for staff to adapt can be under-estimated and under-resourced. As Laurillard (2001) argues, innovation in course design is conditional on staff development and upskilling; new kinds of pedagogy require new knowledge.

Effective professional development balances equipping lecturers with basic technical skills with strategies to integrate technology (Thurlow 1999). Lecturers' diverse technology backgrounds need to be considered, and individualised professional development plans provided (Thurlow 1999). Such individualised professional development plans can be supported through mentor-schemes (Fleming 1999; Sugar 2005; Thurlow 1999). Recently, 
researchers argue that professional development of educators requires situative professional development to promote technology integration (Hughes and Ooms 2004; Holmes 2004; Putnam and Borko 2000; Swan et al. 2000; Zhao et al. 2002). According to Putnam and Borko (2000) there are three conceptual themes central to situative learning:

(1) cognition is situated in particular physical and social contexts;

(2) it is social in nature; and

(3) knowledge is distributed across the individual, others, and tools.

In higher education, such professional education is important for academics and for the technological staff who support them. Appropriate technological and pedagogic training and education for e-developers is also important (Hannon 2008; Oliver et al. 2004; HEFCE 2005).

A study was undertaken in 2004-06 of the work of the learning technologists at an educational technology unit at a university in Australia who supported academic staff in their e-learning developments (Hannon 2008). This was a limited, small study and it seemed useful to undertake a larger study at a university which had a similar central educational development unit and who provided e-learning support to academics.

\section{The case study}

\section{Context}

The decision to purchase and support Blackboard as the university Virtual Learning Environment (VLE) was made in July 2000. At the same time the first institutional Learning and Teaching Strategy was developed which contained targets for the implementation, use and support of the institutional VLE. Staff development was seen as a crucial element to the successful implementation of the VLE and this was a priority for the educational technology unit, which had been set up in May 2000. Seven faculty-based educational technology leaders were therefore appointed that year and seconded for $40 \%$ of their time to roll out the use of the VLE and carry out the necessary staff development within their faculties.

An evaluation conducted by the university (Linsey, Katsifli, and Gipps 2005) showed that by 2005, 2000 modules were available in Blackboard with some level of online support at the university, that $99 \%$ of the students were using Blackboard at least once a week and $88 \%$ of them felt that Blackboard had made a significant contribution to their studies. However, further evaluation revealed that the use of Blackboard had not affected faculty members' overall approach to teaching and that staff were using the system primarily with existing teaching materials. It was therefore proposed that further development was needed to encourage staff to address pedagogical issues and improve blended learning.

In September 2005, three e-developers were appointed for one year to support the integration of blended learning with this resource shared equally between faculties. The term 'e-developer' was chosen by the university and is equated to the role of learning technologist as described by Oliver (2002). The e-developer role involved engaging in a range of activities including:

- staff development in both the pedagogic and technical aspects of the VLE;

- pedagogical support and advice to academic staff; and

- technical support. 
The e-developer met regularly with individual academics or module teams to convert modules that were traditionally delivered face-to-face into blended learning modules combining some aspects of face-to-face delivery and a significant amount of online learning activities.

The objectives of the first year of this programme were to support the development of sustainable e-learning content resources and activities across faculties, to develop strategic exemplars and to provide a model for faculties in terms of their own blended learning strategies. The term blended learning can refer to a number of activities where a range of teaching and learning approaches are employed in the delivery of education. For this case study, the term refers to replacing a certain amount of face-to-face, largely didactic classroom activities with a range of interactive, constructivist online learning activities using the university's VLE. It was hoped that the modules developed would serve as catalysts for development of additional blended learning modules by faculties, who would then fund their own academic learning resource developers. The academic team would provide the subject specialist content and work collaboratively with the e-developers to reach a consensus about design, technological development, and pedagogical approach. Educational technology leaders also had a role, which was to help identify projects, coordinate projects, set up meetings, act as point of contact in the faculty, and follow up issues of concern.

Faculties were invited to select a project that they deemed most suitable for conversion into a blended learning format. The project had to meet criteria which were: the project needed to include both content and activities; it should have executive support; and there should be agreement to sustain the project for two years. It was agreed that each faculty would effectively receive $50 \%$ of an e-developer's time. However it was made clear that they were a central team as the Academic Development Centre (ADC) wanted them to focus on the project aims of improving blended learning, and creating innovation. It was also hoped that the modules would serve as catalysts for development of additional modules by faculties, who would then fund their own academic learning resource developers.

\section{Theoretical background}

The theoretical underpinning for this case study is based upon Land (2004), who examined what educational developers see as the main issues that need to be addressed in their work and the impact of educational context and culture on their practice.

Land (2004) found that an issue for these developers is how change in learning and teaching can be made in organisations that have well-established academic cultures and practices and which may have conflicting priorities.

In planning the case study presented here, the researchers set out to uncover not only what the educational developers saw as their role within the organisation (as Land did), but also how others, especially academic staff who worked with these developers, saw the role of educational developers within the academic community and the university as an organisation.

\section{Methodology}

In 2006, the university's Academic Development Centre (ADC) was asked to carry out an evaluative research project to assess the effectiveness of the 'e-developer model' in terms of engagement of staff with blended learning, dissemination of good practice and the sustainability of the projects. The study was designed to examine how the e-developers worked collaboratively with academic staff, and also the perceptions of the e-developer 
model held by academic staff, e-developers themselves and educational technology leaders in each faculty.

The model of evaluation that was adopted was that of realistic evaluation (Pawson and Tilley 1997). Realistic evaluation follows a 'realist' ontology, objective epistemology, and naturalistic sets of methodologies (as opposed to experiment or quasi-experiment). The basic postulate of 'realistic evaluation' is that

programs work ('have successful outcomes') only in so far as they introduce the appropriate ideas and opportunities ('mechanisms') to groups in the appropriate social and cultural conditions ('contexts'). (Pawson and Tilley 1997, 57)

The aim of 'realistic evaluation' is to give a detailed account of what worked, for whom, and under what circumstances.

The evaluation was therefore grounded in the perspectives of the participants in the programme. In other words, the judgements of how well the programme fared in terms of engagement, influence, and sustainability, and the criteria used to make these judgements, were those of the programme participants. The evaluation therefore explored the views of the principal groups involved in the programme, namely: course teams, educational technology leaders, the e-developers and the strategic leads within the ADC.

Four main areas for exploration were identified from the literature, in discussions with strategic ADC staff and from the e-developer programme documentation. These areas were: engagement, styles of working, influence, and sustainability.

- Engagement (how have staff engaged with the projects?);

- Styles of working (what styles of working 'worked' across the different projects/ faculties? What has been the role of the educational technology leaders?);

- Influence (how influential have the e-developer projects been in the faculties?);

- Sustainability (how sustainable are the processes initiated by the e-developers? Are the 'e-developer resources' being used and developed by staff?).

To understand the perspectives of participants in the e-developer projects, a qualitative evaluation method was chosen. The specific choice of method was guided primarily by the anticipation of the richness (or otherwise) of the data that interviews with different groups yield. The interviews were therefore semi-structured, with questions asked around the main areas identified above.

Interviews were carried out with the Associate Head of ADC, course team members ( $n=19$, typically in pairs), a group interview with the educational technology leaders $(n=7)$ and a group interview with the e-developers $(n=3)$. Interview data were then entered in the qualitative data analysis software, NVivo, to identify themes arising from the data.

\section{Results and discussion}

The analysis focused on the four identified themes of engagement, styles of working, influence, and sustainability, per blended learning project.

\section{Engagement}

Engagement during the project (i.e. regular meetings between the course team and the edevelopers) was a major factor contributing to the success of the projects. Projects during 
which people were more engaged were more successful and were perceived as being more enjoyable and more influential. Crucial factors mentioned by course developers that fostered their engagement during the projects were the personalities of the e-developers, involvement of the educational technology leaders, and feedback from the students.

Motivation for engaging in a blended learning project varied across the different faculties. Motivation can be categorised into intrinsic and extrinsic motivation. Different kinds of motivation for engaging in a blended learning project, intrinsic or extrinsic, did not seem to have had an influence on the perceived success of the project.

The majority of academic staff agreed that the e-developers' involvement in the projects made a difference both to the engagement of staff directly involved and those not involved with blended learning. One stated, 'Staff involved with the project appreciated the time and professional results from the e-developer's input'.

Strategic staff agreed that the e-developers' involvement in the projects made a difference to how staff directly involved in the projects think about their teaching but were neutral about this impact for staff not directly involved with the projects. One staff member said that:

Staff are becoming aware of the potential and many will incorporate material into teaching programmes incrementally. The availability of e-developers enabled staff to begin to think more strategically about blended learning. It is important this support is available consistently and not on a 'fragmented' basis. E-developers gave a useful independent (student?) perspective of material.

\section{Styles of working}

Although opinions about what worked well were sometimes contradictory among the staff members in the different faculties, there was some agreement on the positive characteristics of the programme. The involvement of the educational technology leaders was mentioned as important for several of the projects. However, this was not a crucial component for the success of all projects. For example, for one very successful e-learning project there had been little involvement of the educational technology leader. The support of the Associate Head of ADC was mentioned as crucial for one project. Additionally, seeing what works and showcasing projects within the faculties were mentioned as approaches that worked well to create an interest in the faculties among people who were initially not involved.

Most respondents generally agreed, however, that the positive elements of the programme revolved around the e-developers' characteristics. Appreciated were their:

- 'flexibility and availability'

- 'reliability and efficiency'

- 'technical knowledge'

- skill at passing on 'ideas about the best ways to deliver the materials'

- ability to 'absorb the content of the modules'

- 'provision of right solutions'

- 'encouragement'

- 'talent, creativity and enthusiasm'

- 'sensitivity to the uniqueness of schools/faculties'

- 'good response time'

- 'comfortable manageable style of working'

- presentation of 'interesting alternatives to conventional quizzes' 
Overall, the e-developers appeared to act as role models/mentors for e-development and were given considerable credit for the success of the individual projects:

Without them - the e-developers - I wouldn't have been able to move forward with this project.

The e-developer programme was also described by academic staff as 'a catalyst' and 'speeded up things', according to one academic staff member.

Preferences in communication methods between the e-developers and the course team members varied. The communication method preferred by most participants was face-toface meetings. A few people commented negatively about the efficiency of communication by e-mail due to slow response times of the e-developers, whereas this communication method worked well for others.

Three areas of debate emerged related to styles of working. One issue was about whether the e-developers should be based at the central ADC, or within their designated faculty. Another issue was about working with individual members of staff versus working with a team of course developers. The third issue was about the role and input the educational technology leaders had in the projects. Some educational technology leaders were more involved than others and quantity and quality of involvement varied greatly. Some educational technology leaders were not involved at all. One member of staff said that the educational technology leaders informed them about the project but '... did no more than that'.

Other educational technology leaders were very involved and worked together with the course developer as a team. Although the educational technology lead was the first port of call or link, some were only marginally involved in the projects.

Additionally, staff members were unclear about the role of the educational technology leaders:

It took me a while to work this out, that he was the person to approach for help with blended learning in the faculty...

If I had known he was in charge, I would have had fewer scruples to ask him and bother him.

Other factors also undermined the success, and even prevented the progress of the projects. These included scepticism towards blended learning, or misunderstandings about what blended learning is. However, the main undermining factors were lack of time and unclear expectations. Every faculty member involved in a blended learning project said they underestimated the time involved in the project.

It requires immense time of preparation to provide materials to e-developers.

Some projects also progressed slowly because academic staff did not provide materials to e-developers on time. Academic staff reported that they are 'burdened with other things'. Several mention the idea of giving release time for academic staff involved in development. A factor that limited the success of one project was the perceived late announcement of the blended learning project, which prevented one faculty to adequately plan:

It was not given adequate planning time with respect to academics' time. Academics' timetables are already set for next year and once set they are relatively inflexible.

There were several issues that caused problems at the beginning stage of the projects. The e-developers overestimated academic staff members' skills and knowledge of technology. One staff member reported: 
At the beginning it was quite difficult for me to get into it, the problem was that they (the e-developers) assumed too much knowledge from me, that I knew about blended learning.

There was also a problem of unclear expectations where academic staff hoped that the e-developers would:

add interactive in addition to static material (but) ... We had to do it on our own.

If e-developers had clarified from the beginning what was expected from us that would have made it easier for us.

This understandably contributed to misconceptions about responsibilities and workload. Likewise, there was little clarity among the academic staff about the ability, commitment, time availability and organisational structure of the e-developers' roles. One of the academic staff members said:

I wanted them to develop it ... [I gave the e-developer] a pile of things to read ... I have not seen her since ... there was no clarity as to precisely what their ability might be ... I don't think we knew precisely what their role was either.

Staff agreed that the way the e-developers collaborated with staff on the projects was successful in helping meet the aims of the projects. One member of staff said:

There was good rapport between e-developers and faculty staff. Progress between meetings was significant and the meetings permitted evaluation and updating of project implementation.

\section{Influence}

With the exception of one project, at the time of the evaluation it was too early to tell if, and to what extent, the blended learning project had had an impact on student learning. This will be the subject of a further evaluative project. However, most people involved believed in the potential of that impact.

The project has had an impact on the modules:

It raises the level of our materials by adding to the resources.

The module was improved through this format.

One member of staff said that the impact was great because external colleagues are now purchasing their blended learning product. One particular blended learning module was subsequently implemented in several other courses so those courses could also benefit from the project.

The project also had an impact on staff, including:

- raised awareness;

- fostered communication among staff, including cross-faculty/cross-discipline; and

- increased interest among staff who were not directly involved.

Staff also indicated that they were starting to think and talk about applying blended learning strategies to other modules. Presentations about the projects had been attended by staff with 
much interest and some of the staff involved had been contacted by staff not currently involved to talk about potential blended learning projects. One academic reported that a colleague said, 'Oh, I want to do that, oooh, that is good'.

One course was implemented whilst still in the process of being created. Academic staff working on this particular project received verbal and non-verbal feedback from students and they reported the following impressions they had:

- Students worked harder on this module because they had to keep up with the online testing;

- Students enjoyed the variety of formats;

- Students were more positive about the content than previously;

- Students booked video rooms to view videos together, a sign of more collaborative learning among the students;

- The blended learning component helped students with time management because in order to meet the deadlines, they had to plan their time more efficiently;

- Student performance improved from the previous year.

One academic mentioned that she is thinking of developing her own technical skills now because she does not want to be dependent on e-developers. However, she realises that she will need technical assistance for a while before she can do this.

Perceptions about the influence of the projects varied among the strategic faculty staff. One faculty staff member said:

There is the potential to be influential. The ... project has had a very positive impact. It's ease of use and obvious use as a teaching resource has shown how blended learning can have huge added value. The skills resource is very much 'work in progress' on an ambitious project, and ongoing support is essential for it to have general influence.

One final area of influence was in the mentorship role played by the e-developers, serving as a guide/role model for good pedagogic practices through the technology:

As a model of what can be achieved, this ... has provided both inspiration and insight into the use of blended resource materials. It is hoped that the sustainable nature of the project will allow dissemination of the approach across the whole faculty.

\section{Sustainability}

Sustainability was a major concern for all people involved. All academic staff believed that the blended learning components could not be sustained without the e-developers. Most people expressed concerns about availability of support and lack of time and knowledge of staff.

(Blended learning) will stall without e-developer input ... it takes significant time to maintain, more than a face to face mode.

They also expressed concerns about updating/upgrading the current modules because the shelf-life of materials varies and staff turnover was a concern, resulting in different teaching styles and different approaches to the subject. Furthermore, multimedia resources age very quickly. Concerns about sustainability had an impact on the effort made towards these projects. One person reported: 
Why should we bother to produce a project that isn't going to be resourced at all?

Lack of time is also an obstacle for further development of existing project or for development of new projects: However, others stated:

The use of university-based e-developers has advantages (more people equals a broader range of expertise) and disadvantages (there are conflicting demands on their time). The faculty has (as a result of this project) requested funding to have a faculty-based e-developer alongside the university provision. This will broaden the range of support available and provide dedicated staff for maintenance of interface to the material and content.

Overall, academic staff were extremely positive about their experience with their work with the e-developer. Staff described it as a learning experience and identified the value of the enthusiasm and fun that the projects generated. The e-developers were described as good role models. However, some unanticipated aspects of the blended learning projects were identified, including the need for the projects to be better resourced and more tightly structured, that the work was slower than was hoped and that, although pleased with the end results, there was more work for academic staff than they had anticipated.

\section{Conclusion}

This paper has explored the introduction of technology in higher education, and the way in which roles and practices influenced this process. The findings suggest that the characteristics of e-developers that are associated with success of the projects are their engagement with academic staff, and their enthusiasm and pedagogic and technical skill. The involvement of the educational technology leaders, though valued where it did take place, was not a crucial component of success in all cases.

Crucial factors mentioned by course developers that fostered their engagement during the projects were the personalities of the e-developers and feedback from the students. The success factor that most respondents agreed on was that the programme revolved around the e-developers' positive characteristics and the e-developers were given considerable credit for the success of the individual projects.

The most common reasons that limited, or prevented, the completion of projects were lack of communication, differing expectations and misconceptions about the knowledge and skills of the e-developers, and scope of the project itself. Other issues that impeded the development of the projects were academic staff not providing materials to e-developers on time; e-developers overestimating staff members' skills and knowledge of technology; and misconceptions about responsibilities and workload. Additionally, more general factors that undermined success included scepticism towards blended learning and misunderstandings about what blended learning is.

Although at the time of the evaluation it was too early to tell if, and to what extent, the blended learning project had had an impact on student learning, most people involved were positive about the potential of that impact.

Sustainability of these initiatives is a major concern for all people involved. All participating academic staff members believe that these blended learning initiatives cannot be sustained without the e-developers. Most people also expressed concerns about availability of support and lack of time and knowledge of staff if these projects are left to faculties to develop.

This research project has supported the importance of staff development emphasised in the literature. However, it offers an alternative organisational perspective and model of 
development that does not involve taking academic staff out of the teaching area to provide this development. The e-developers can provide development within the context that academics are working in on real problems, thereby supporting situative professional development, which helps promote technology integration into teaching. In addition, the e-developers provided cost-effective mentorship which participants believed would have a positive impact on student learning.

\section{References}

Adamy, P. 2000. The influence of organizational culture on technology integration in teacher education. Paper presented at the annual meeting of the American Educational Research Association, April 24-28, in New Orleans.

Bates, A.W. 2000. Managing technological change. Strategies for college and university leaders. San Francisco: Jossey-Bass.

Borko, H. 2004. Professional development and teacher learning: Mapping the terrain. Educational Researcher 33, no. 8: 3-15.

Cathings, M.H., and S.K. MacGregor. 2000. Professional development models in action: Supporting teachers in technology implementation. Paper presented at the annual meeting of the American Educational Research Association, April 24-28, in New Orleans.

CEO Forum on Education and Technology. 2001. School technology readiness report: Key building blocks for student achievement in the 21st century. The CEO Forum on Education and Technology, year four. Washington, DC: CEO Forum. http://www.ceoforum.org.

Chuang, H., A. Thompson, and D. Schmidt. 2003. Faculty technology mentoring programs: Major trends in the literature. Journal of Computing in Teacher Education 19, no. 4: 101-6.

Cole, K., M. Simkins, and W. Penul. 2002. Learning to teach with technology: Strategies for in-service professional development. Journal of Technology and Teacher Education 10, no. 3: 431-55.

Cuban, L. 2000. So much high-tech money invested, so little use and change in practice: How come? Paper prepared for the Council of Chief State School Officers' annual Technology Leadership Conference, February 20-22, in Washington, D.C.

Cuban, L., H. Kirkpatrick, and C. Peck. 2001. High access and low use of technologies in high school classrooms: Explaining an apparent paradox. American Educational Research Journal 38, no. 4: 813-34.

Fleming, D.S. 1999. New directions in professional development. http://www.education.umn.edu/ CAREI/Reports/Rpractice/Spring96/directions.html.

Hannon, J. 2008. Doing staff development: Practices, dilemmas and technologies. Australasian Journal of Educational Technology 24, no. 1: 15-29.

Higher Education Funding Council for England (HEFCE). 2005. HEFCE strategy for e-learning. Statements of Policy March 2005/12. http://www.hefce.ac.uk/pubs/hefce/2005/05.

Holmes, A. 2004. Envisioning, understanding, and encouraging technology integration: Case studies examining professional development and technology integration. Dissertation, University at Albany, SUNY.

Hughes, J.E., and A. Ooms. 2004. Content-focused technology inquiry groups: Preparing urban teachers to integrate technology to transform student learning. Journal of Research on Technology in Education 36, no. 4: 397-411.

Kenny, J. 2002. Managing innovation in educational institutions. Australian Journal of Educational Technology 18, no. 3: 359-76.

Land, R. 2004. Educational development: Discourse, identity and practice. Maidenhead: Open University Press.

Laurillard, D. 2001. The E-University: What have we learned? The International Journal of Management Education 1, no. 2: 3-7.

Linsey, T., D. Katsifli, and C. Gipps. 2005. The costs and benefits of implementing a universitywide VLE: some real data. Journal of Science Education 6: 27-9.

Norum, K., R.S. Grabinger, and J.A. Duffield. 1999. Healing the universe in an inside job: Teachers' views on integrating technology. Journal of Technology and Teacher Education 7, no. 3: 187-203.

Oliver, M. 2002. What do learning technologists do? Innovations in Education and Teaching International 38, no. 4: 245-52. 
Oliver, M., R. Sharpe, J. Duggleby, D. Jennings, and D. Kay. 2004. Accrediting learning technologists: A review of the literature, schemes and programmes. ALT Accreditation Project Report 1 , February.

O’Neill, K., G. Singh, and J. O’Donoghue. 2004. Implementing elearning programmes for higher education: A review of the literature. Journal of Information Technology Education 3: 313-23.

Pajo, K., and C. Wallace. 2001. Barriers to the uptake of web-based technology by university teachers. Journal of Distance Education 16, no. 1.

Pawson, R., and N. Tilley. 1997. Realistic evaluation. London: Sage.

Polselli, R. 2002. Combining web-based training and mentorship to improve technology integration in the k-12 classroom. Journal of Technology and Teacher Education 10, no. 2: 247-72.

Putnam, R., and H. Borko. 2000. What do new views of knowledge and thinking have to say about research on teacher learning? Educational Researcher 29, no. 1: 4-15.

Sugar, W. 2005. Instructional technologist as a coach: Impact of a situated professional development program on teachers' technology use. Journal of Technology and Teacher Education 13, no. 4: $547-71$.

Swan, K., J.E. Bowman Jr., A. Holmes, J.D. Vargas, and J. Richardson. 2000. Technology, professional development and the culture of the schools. Paper presented at the annual meeting of the American Educational Research Association, April 24-28, in New Orleans.

Swan, K., A. Holmes, J.D. Vargas, S. Jennings, E. Meier, and L. Rubenfeld. 2002. Situated professional development and technology integration: The Capital Area Technology and Inquiry in Education (CATIE) mentoring program. Journal of Technology and Teacher Education 10, no. 2: 169-90.

Thurlow, J.P. 1999. Teachers as technologists: Professional development for technology integration. Paper presented at the Annual Meeting of the International Reading Association, in San Diego, CA.

UCISA (Universities and Colleges Information Systems Association). 2006. HEITS 2005. Higher Education Information Technology Statistics (HEITS). http://www.ucisa.ac.uk/activities/stats/ stats05.htm.

Zhao, Y., K. Pugh, S. Sheldon, and J. Byers. 2002. Conditions for classroom technology innovations. Teachers College Record 104, no. 3, 482-515. 